University. The IHE, whose name was soon changed to the Institute for Ageing and Health, was founded on a platform of excellence in geriatric medicine and in dementia research. Its expansion after 1999 to include a major programme on the biology of ageing has led to the creation of a substantial, multidisciplinary research environment which includes biologists, clinicians, technologists and social scientists. Funding was quickly obtained for a new building, the Henry Wellcome Laboratory for Biogerontology Research, completed in 2003. This has been followed in rapid succession by four further buildings which together began the formation of an integrated biomedical Campus for Ageing and Vitality. In addition to new buildings, expansion of research was made possible with research funding from the BBSRC to create the Centre for Integrated Systems Biology of Ageing and Nutrition, spearheading the study of complexity in ageing ${ }^{15}$; the Wellcome Trust for the Clinical Ageing Research Unit directed by David Burn; the UK Research Councils Lifelong Health and Wellbeing initiative for the Centre for Brain Ageing and Vitality directed by Doug Turnbull; and the National Institute for Health Research for the Biomedical Research Centre on Ageing directed first by Chris Day and now by Patrick Chinnery. At the same time, there was opportunity to influence the national agenda for ageing research as specialist adviser to a House of Lords Science and Technology Select Committee inquiry into ageing in 2005 and through a Government Office for Science Foresight report on Mental Capital Through Life. ${ }^{16,17}$ In February 2010, Newcastle University was awarded the Queen's Anniversary Prize for Higher and Further Education for its programme of research on ageing and health.

\section{Future prospect}

Research on the underlying mechanisms of ageing is now a major discipline within the life sciences, recognised as a priority by most of the major funding agencies. The frequency with which research articles appear in the highest impact general scientific and medical journals is a clear sign of the transformed status of the field. Nevertheless, immense challenges are still to be addressed, including the mechanistic complexity of the ageing process, its diversity of effects in cells and tissues, the genetics of longevity, and the fascinating relationship between intrinsic ageing and the many diseases to which it gives rise. It has been exciting to have experienced this transformation in research on ageing from its days as a scientific rarity to one of the biggest growth areas in biomedical science. It is about time.

\section{References}

1 Kirkwood TBL, Holliday R. Commitment to senescence: a model for the finite and infinite growth of diploid and transformed human fibroblasts in culture. J Theor Biol 1975;53:481-96.

2 Holliday R, Huschtscha LI, Tarrant GM, Kirkwood TBL. Testing the commitment theory of cellular ageing. Science 1977;198:366-72.

3 Kirkwood TBL, Holliday R. The stability of the translation apparatus. J Mol Biol 1975;97:257-65.

4 Kirkwood TBL. Evolution of ageing. Nature 1977;270:301-4.
5 Kowald A, Kirkwood TBL. Towards a network theory of ageing: a model combining the free radical theory and the protein error theory. J Theor Biol 1994;168:75-94.

6 Kowald A, Kirkwood TBL. A network theory of ageing: the interactions of defective mitochondria, aberrant proteins, free radicals and scavengers in the ageing process. Mut Res 1996;316:209-236.

7 Kirkwood TBL. Repair and its evolution: survival versus reproduction. In: Townsend CR, Calow P (eds), Physiological ecology: an evolutionary approach to resource use. Oxford: Blackwell Scientific Publications, 1981:165-89.

8 Kirkwood TBL. Comparative and evolutionary aspects of longevity. In: Finch CE, Schneider EL (eds), Handbook of the biology of aging. New York: Van Nostrand Reinhold, 1985:27-44.

9 Kirkwood TBL, Cremer T. Cytogerontology since 1881: a reappraisal of August Weismann and a review of modern progress. Hum Gen 1982;60:101-21.

10 Comfort A. Test-battery to measure ageing-rate in man. Lancet 1969;27:1411-5.

11 Kapahi P, Boulton ME, Kirkwood TBL. Positive correlation between mammalian life span and cellular resistance to stress. Free Radical Biology Med 1999;26:495-500.

12 Schächter F, Cohen D, Kirkwood TBL. Prospects for the genetics of human longevity. Hum Gen 1993;91:519-26.

13 Schächter F, Faure-Delanef L, Guénot F et al. Genetic associations with human longevity at the APOE and ACE loci. Nature Gen 1994;6:29-32.

14 Westendorp RGJ, Kirkwood TBL. Human longevity at the cost of reproductive success. Nature 1998;396:743-6.

15 Kirkwood TBL. A systematic look at an old problem. Nature 2008;451:644-7.

16 Beddington J, Cooper CL, Field J et al. The mental wealth of nations. Nature. 2008;455:1057-60.

17 Kirkwood TBL, Bond J, May C, McKeith I, Teh M. Foresight Mental Capital and Wellbeing Project. Mental capital through life: future challenges. London: Government Office for Science, 2008.

\section{Sarcopenia and some simple approaches to modifying the consequence of ageing}

Sarah (Sallie) Lamb, professor of trauma rehabilitation, Nuffield Department of Rheumatology and Orthopaedic Surgery, University of Oxford and director and professor of rehabilitation, Warwick Clinical Trials Unit, University of Warwick

Email: S.Lamb@warwick.ac.uk

In popular culture, and from the time of the ancient Greeks, aging has traditionally been associated with declining physical and cognitive vitality. ${ }^{1}$ The size and profitability of the 'antiageing' industry indicates the desire of many people to stave off the apparently inevitable cosmetic and functional insults of time. Potential remedies range from creams, elixirs, vitamin tonics and supplements, to the fountain of youth portrayed in the 1980s film Cocoon, in which the residents of a retirement village sample the delights of rejuvenation. The emerging culture of celebrity in the 21st century provides glowing examples of older actors, actresses and so on, who attest to the power of diet, exercise and a handy cosmetic surgeon. Such experiences of aging are by no means universal, but open a portal to understanding how progress in exercise physiology and prescription over the last 20 years provide real hope of enhancing the experience of aging. 
The role of muscle and neuromuscular function to health and functioning in later life is one of the most important concepts to have been established in the last 20 to 30 years. ${ }^{1}$ Observational studies revealed how people build muscle strength over the first two decades of life, with decline starting around the third and fourth decade and, finally, decline accelerates from the sixth decade. ${ }^{2}$ These early observations of age-associated declines in strength led to closer scrutiny of changes in the structure and function of muscle. Ensuing investigations were to link ageassociated decline in muscle mass (sarcopenia) with waning strength. ${ }^{3}$ However, the relationship between strength and mass was not entirely linear. A gradual process of muscle fibre apoptosis and subsequent scattered reinnervations across disparate fibres means that muscles can no longer generate force in a smooth and graded fashion. Some types of muscle fibre atrophy completely in older age, and there is a reduction in the number of spinal motor neurones. An increase in fat infiltration, a shift from anabolic to catabolic protein synthesis, and decreased oxidative capacity also add to a decline in the amount of force that can be generated per unit of cross-sectional area. ${ }^{4}$ The impact of these changes is evident particularly on the decline of speed with which force can be produced (muscle power); this is estimated to be $3.5 \%$ per annum from the age of 65 years onward, and is substantially greater than the loss in strength alone. ${ }^{5}$

The ability to thrive depends on a range of basic functions including mobility, standing, seeing, hearing and perception which, when integrated, allow complex tasks to be undertaken. In 1986, Young was one of the first gerontologists to suggest that basic motor tasks, for example rising from a chair, require a minimum fixed threshold of muscular strength and power to complete. ${ }^{6}$ Importantly, he suggested that the strength threshold for a specific task is a fixed entity. Researchers had begun to observe that muscular strength declines with age, and that against this backdrop, it was possible that strength could drop below the threshold required for particular tasks, and that people become disabled as a consequence. This mechanism is particularly potent in catastrophic health insults such as hip fracture. ${ }^{2}$ Loss of muscular mass and function also plays a critical role in the genesis of frailty and falls and, possibly, other linked geriatric syndromes including depression and cognitive impairment. While the links between sarcopenia and immobility are easy to conceptualise, the links to falls are perhaps less clear. Reference to standard anatomy textbooks describe postural and motor muscles, and emphasise the importance of postural muscles in stabilising the skeleton and body mass within the base of support while allowing prolonged standing, reaching, bending and so on. Other muscles, particularly those around the ankle, perform important functions in ensuring that perturbations of the centre of mass are corrected rapidly, and falls prevented. ${ }^{7}$

Muscle is the largest organ in the body and plays an important role in providing a reserve of protein vital to ensuring survival during periods of illness and threat. ${ }^{2}$ Mid-life hand grip strength is an independent and strong risk factor for time to death, ${ }^{8}$ most likely reflecting the reserve that people have to draw on in times of physiological stress and illness. ${ }^{8}$ Research over the last 10 years has focused on establishing potential pathways to sarcopenia, with pro-inflammatory cytokines, reactive oxidative species, and various pathways to altered protein synthesis being explored as potential mechanisms that might be targeted by pharmacological intervention. ${ }^{2,9}$

The answer may, thankfully, be simpler. An extension of the theory of age-associated decline came from Edwards and Larsen, ${ }^{10}$ who highlighted the wide variation in the trajectory of age-associated decline in function, and suggested that an element of decline was attributable to physical inactivity, and the effects of acute and chronic illness. Over the last two decades, numerous randomised controlled trials have established that progressive resistance training in older people improves muscle mass, strength and function. ${ }^{11}$ Strength training increases the reserve of available muscle mass and function in everyday life. However, it is unclear whether the underlying rate of sarcopenia is modified. ${ }^{2}$ The LIFE study funded by the National Institutes of Health will examine the hypothesis that strength and mixed training can be used to retard functional decline and to determine whether the intervention is sufficiently potent to impact on important public health outcomes including compression of morbidity, transition to long-term care and mortality. ${ }^{12}$ The therapeutic effects of strength training are thought to extend to fibre hypertrophy and enhanced stimulation of spinal alpha neurones. ${ }^{4}$ An important and unresolved question is whether increasing the amount of day-to-day activity, such as walking, house cleaning, and so on, is sufficient to stimulate an increase in muscle mass and function. ${ }^{2}$

The benefits of strength training extend across a range of health conditions. Although the mechanism by which sarcopenia and declining muscular function induce depression and cognitive impairment are unclear, strength training raises homocysteine and insulin like growth factor ${ }^{1}$ and these effects, along with increased heart rate and oxygenation, appear to mediate therapeutic effects. ${ }^{12,13}$ Exercise increases the volume of the anterior horn of the spinal cord, which might play a role in dementia. ${ }^{14}$ Strength training and other novel interventions, such as electrical stimulation, increase muscle bulk and function after hip fracture, and contribute to regaining an equal proportion of muscular mass in both legs which is an essential step in recovery. ${ }^{15}$ Pain is an important pathway to reduced muscular function, and is potentially a modifiable risk factor with broad public health relevance given the prevalence of osteoarthritis and musculoskeletal pain in the aging population. ${ }^{16}$

My career has focused on contributing to, and drawing from, this emerging epidemiological and scientific base, and transforming it into practical interventions that can be, and are being, tested in the NHS in the context of large, pragmatic trials. By conducting large trials, removing the traditional and unjustified upper age limit seen in many trial designs, and developing ethical approaches to inclusion of people with significant comorbidity and cognitive impairment, we are developing a solid evidence base to guide clinical practice. For example, in a trial of 
700 people, older adults benefit just as well from cognitive behavioural interventions to promote exercise and activity despite lower back pain, as do their younger counterparts. ${ }^{17}$ Multifactorial interventions that include the option to exercise in nursing homes are effective in reducing falls, frailty and with encouraging evidence for hip fracture prevention. ${ }^{18}$ In the next year we will report a trial of over 1,000 older people living in nursing home accommodation, which has investigated the clinical and cost effectiveness of group exercise interventions and staff training to prevent and manage depression. ${ }^{19}$ We will be studying the effect on linked geriatric syndromes and generic health-related quality of life with interest. Many more important trials are in development, notably, a trial of exercise interventions to manage mild to moderate dementia, a condition for which there is a paucity of treatment options. We have investigated the effects of providing exercise in different formats, such as health walks which utilise the natural resource and enjoyment of the countryside. ${ }^{20}$

These trials have highlighted the realities of everyday public health and health service provision and the challenges of encouraging regular participation in exercise. While cohort effects were considered a potential explanation for poor participation of older people, ${ }^{21}$ the effects of modern culture seem set to intensify sedentary behaviour. The metabolic consequence of a shift from anabolic metabolism to catabolism may provide explanations as to why some older people find exercise uncomfortable. Intercurrent illness is now an established reason why many older people fail to engage with exercise programmes in the long term. ${ }^{2}$ Pain, fatigue, lack of familiarity with exercise, and an increasing sense of vulnerability should also be considered. Finally, an evolutionary perspective provides an interesting new approach. ${ }^{22}$ Humans have evolved quickly to the point that the link between foraging, movement and survival has been broken. The pathway to increased physical activity is set to operate at a much higher level of sophistication. Individuals need to understand the benefits, and to make informed choices about building their internal pension for a healthy older age. The NHS and other global health systems must learn best how to help people in this goal, and to invest money wisely to support the endeavour.

\section{References}

1 Ferrucci L, Studenski S. Diabetes, muscles, and the myth of Ulysses' Bow. Diabetes Care 2009;32:2136-7.

2 Buford TW, Anton SD, Judge AR et al. Models of accelerated sarcopenia: Critical pieces for solving the puzzle of age-related muscle atrophy. Ageing Research Reviews 2011.

3 Cruz-Jentoft AJ, Baeyens JP, Bauer JM et al. Sarcopenia: European consensus on definition and diagnosis: Report of the European
Working Group on Sarcopenia in Older People. Age Ageing 2010;39:412-23.

4 Narici MV, Maffulli N. Sarcopenia: characteristics, mechanisms and functional significance. Br Med Bull 2010;95:139-59.

5 Skelton DA, Greig CA, Davies JM, Young A. Strength, power and related functional ability of healthy people aged $65-89$ years. Age Ageing 1994;23:371-7.

6 Young A. Exercise physiology in geriatric practice. Acta Med Scand 1986;711:227-32.

7 Lord SR, Clark RD, Webster IW. Postural stability and associated physiological factors in a population of aged persons. J Gerontol 1991;46:M69-M76.

8 Rantanen T, Volpato S, Ferrucci ML et al. Handgrip strength and cause-specific and total mortality in older disabled women: exploring the mechanism. J Am Geriatr Soc 2003;51:636-41.

9 Stenholm S, Maggio M, Lauretani F, Bandinelli S et al. Anabolic and catabolic biomarkers as predictors of muscle strength decline: the InCHIANTI Study. Rejuvenation Res 2010;13:3-11.

10 Edwards KE, Larson EB. Benefits of exercise for older adults. Clinics Geriatr Med 1992;8:35-50.

11 Liu CJ, Latham NK. Progressive resistance strength training for improving physical function in older adults. Cochrane Database Syst Rev 2003;(2):CD002759.

12 Singh N, Stavrinos T, Scarbek Y et al. A randomized controlled trial of high versus low intensity weight training versus general practitioner care for clinical depression in older adults. J Gerontol Biol Sci Med Sci 2005;60:768-76.

13 Liu-Ambrose T, Donaldson MG. Exercise and cognition in older adults: is there a role for resistance training programmes? Br J Sports Med 2009;43:25-7.

14 Deslandes A, Moraes H, Ferreira C et al. Exercise and mental health: many reasons to move. Neuropsychobiology 2009;59:191-8.

15 Lamb SE, Morse RM, Grimley Evans JG. The relevance of leg extensor power, postural sway and other factors to the recovery of mobility after proximal femoral fracture. Age Ageing 1995;24:308-14.

16 Lamb SE, Guralnik JM, Buchner DM et al. Factors that modify the association between knee pain and mobility limitation in older women: the Women's Health and Aging Study. Ann Rheum Dis 2000;59:331-7.

17 Lamb SE, Hansen Z, Lall R et al. Group cognitive behavioural treatment for low-back pain in primary care: a randomised controlled trial and cost-effectiveness analysis. Lancet 2010;375:916-23.

18 Rapp K, Lamb SE, Erhardt-Beer L et al. Effect of a statewide fall prevention program on incidence of femoral fractures in residents of long-term care facilities. J Am Geriatr Soc 2010;58:70-5.

19 Underwood M, Eldridge S, Lamb SE et al. Protocol for the OPERA trial: a randomised trial of an exercise intervention for older people in residential and nursing accommodation. [ISRCTN No: 43769277]. BioMed Central 2010.

20 Lamb SE, Bartlett HP, Ashley A, Bird W. Can lay-led walking programmes increase physical activity in middle aged adults? A randomised controlled trial. J Epidemiol Comm Health 2002;56:246-52.

21 Grimley Evans J, Goldacre M, Hodkinson ML, Lamb SE, Savory M. Health: abilities and wellbeing in the third age: The Carneghie Inquiry into the Third Age. London: Carneghie Trust, 1992.

22 Eaton SB, Cordain L, Sparling PB. Evolution, body composition, insulin receptor competition, and insulin resistance. Preven Med 2009;49:283-5. 